\section{Least Squares Refinement of the Structure of $\mathrm{Mo}_{17} \mathrm{O}_{47}$}

\section{A R S I H L BORG}

Institute of Chemistry, University of Uppsala, Uppsala, Sweden

A crystal structure determination of the molybdenum oxide $\mathrm{Mo}_{17} \mathrm{O}_{47}$ was reported earlier in this journal ${ }^{1}$. The structure which is rather complicated shows some interesting features; thus molybdenum atoms are coordinated by seven oxygen atoms at the corners of a pentagonal bipyramid in addition to which the separations between some molybdenum atoms are shorter than in the pure metal. The unit cell dimensions reported are $a=$ $21.61_{5} \AA, b=19.63_{2} \AA, c=3.951 \AA$ and the space-group is Pba2 (No. 32).

The metal atom positions were refined by applying back-shift corrections, first to the $x$ and $y$ coordinates in projections along the short $c$ axis and finally to the $z$ coordinates in sections of the three. dimensional electron density function. The oxygen positions were determined from successive difference syntheses in which the calculated contributions from the metal atoms were subtracted.

After these results had been published computer programmes for absorption correction and least squares refinement became available to the author and further refinement of the structure was performed and is reported below.
The intensity data previously recorded were used also for this refinement. Absorption corrections were applied to the reflexions $h k 1-h k 3$ by means of the programme No. 6019 in the World List of Crystallographic Computer Programs, Ist Ed., Sept. 1962. The $h k 0$ reflexions only had previously been corrected for absorption by a graphical method.

The atomic coordinates listed in Table 2 of Ref. ${ }^{1}$ were used as starting parameters for the refinement. This was performed with the aid of a block diagonal matrix programme (assig. ned the number 6023 in the World List) written by S. Åsbrink and C.-I. Brändén and described briefly as a part of a paper recently published in this journal ${ }^{2}$. The structure factors were weighted by Cruickshank's weighting function in the form $w=\left(60+\left|F_{0}\right|+0.006\right.$ $\left(F_{0}{ }^{2}\right)^{-1}$. Weight analyses obtained in the last cycle are given in Table 1 . The atomic scattering curves for $\mathrm{Mo}^{3}+\left({ }^{3}\right)$ and $\mathrm{O}^{-}\left({ }^{4}\right)$ were used.

In the space group $\mathrm{Pba2}$ the origin of the unit cell is not fixed by symmetry in the [001] direction and the $z$ coordinate of atom $\mathrm{Mo}_{1}$ (arbitrarily chosen) was therefore maintained during the refinement at the previously re. ported value of 0.579 .

The refinement was considered to be completed when the successive shifts in the atomic parameters were less than $15 \%$ of the calculated standard deviations; the discrepancy index $R$ decreased from 0.082 to 0.057 . The atomic parameters thus obtained are listed in Table 2. A three-dimensional full-difference synthesis was finally calculated over the unique part of the unit cell. The largest maxima in this function have a magnitude of about $20 \%$ of the mean height of the oxygen maxima in

Table 1. Analysis of the weighting scheme used. $\Delta=\left\|F_{\mathrm{o}}|-| F_{\mathrm{c}}\right\|$

\begin{tabular}{|c|c|c|c|c|c|}
\hline $\begin{array}{c}\text { Interval } \\
\text { sin } \Theta\end{array}$ & $\begin{array}{c}\text { Number of } \\
\text { independent } \\
\text { reflexions }\end{array}$ & $\begin{array}{c}\overline{w \Delta^{2}} \\
\text { (normalized) }\end{array}$ & $\begin{array}{c}\text { Interval } \\
F_{\text {o }}\end{array}$ & $\begin{array}{c}\text { Number of } \\
\text { independent } \\
\text { reflexions }\end{array}$ & $\begin{array}{c}\overline{\Delta^{2}} \\
\text { (normalized) }\end{array}$ \\
\hline $0-0.46$ & 162 & 0.89 & $0-40$ & 0 & \\
$0.46-0.58$ & 127 & 0.89 & $40-80$ & 18 & 0.72 \\
$0.58-0.67$ & 94 & 1.22 & $80-120$ & 162 & 1.15 \\
$0.67-0.74$ & 78 & 1.03 & $120-160$ & 265 & 1.06 \\
$0.74-0.79$ & 59 & 0.98 & $160-200$ & 149 & 0.67 \\
$0.79-0.84$ & 48 & 1.16 & $200-240$ & 76 & 0.85 \\
$0.84-0.89$ & 66 & 1.12 & $240-280$ & 37 & 0.87 \\
$0.89-0.93$ & 53 & 1.06 & $280-320$ & 26 & 0.78 \\
$0.93-0.97$ & 56 & 0.76 & $320-360$ & 16 & 0.94 \\
$0.97-1.00$ & 30 & 1.20 & $>360$ & 24 & 2.59 \\
\hline
\end{tabular}

Acta Chem. Scand. 17 (1963) No. 5 
Table 2. Atomic parameters obtained by the least squares refinement.

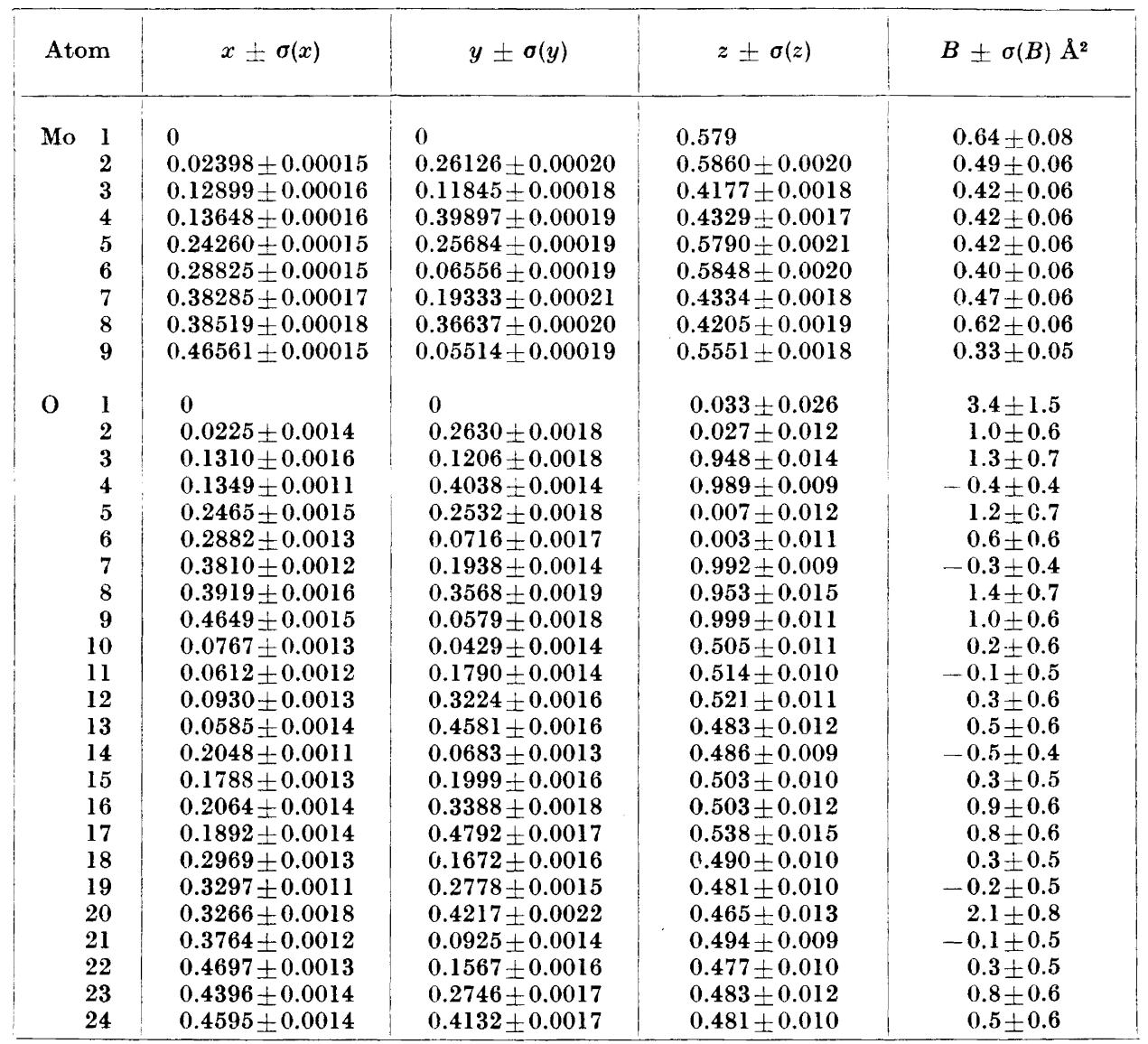

the electron density function and the largest minima a magnitude of less than $30 \%$ of the same value.

As can be seen in Table 2 the values obtained for the isotropic temperature factors $B$ show a large divergence, particularly in the case of the oxygen atoms. Similar effects have been observed during the refinement of other molybdenum oxide structures, for example that of $\mathrm{Mo}_{4} \mathrm{O}_{11}(\mathrm{o}-\mathrm{rh})^{5}$. In the present case the corresponding standard deviations $\sigma(B)$ are also rather large and the deviations from the mean values are scarcely of statistical significance.

The new positions of the molybdenum atoms differ by a maximum of $0.04 \AA$ from those previously given while the corresponding maximum difference for the oxygen positions are $0.35 \AA$. These differences are chiefly due to shifts in the $z$ coordinates and the principal features of the structure, as described and discussed earlier, remain unchanged. Only a few points of interest regarding the interatomic distances will be mentioned here. A comparison of the crystal structures of all molybdenum oxides and their interatomic distances will be published later by the present author.

The most interesting Mo-Mo distance is the short separation between $\mathrm{Mo}_{9}$ and $\mathrm{Mo}_{0}{ }^{\prime}$. This is now calculated to be $2.626 \AA$ (with a standard deviation of $\pm 0.007 \AA$ ) only very slightly different from the previous value of $2.639 \AA$. This distance should be compared with the interatomic distance of $2.725 \AA$ found in the pure metal. 
The distortion of the coordination around the molybdenum atoms arises mainly from displacements of the metal atoms from the centres of the polyhedra (octahedra or pentagonal bipyramids) in the $+z$ or $-z$ direction towards the oxygen atoms at the apices. Accordingly, these Mo-O distances are short, the mean value being $1.764 \pm 0.017 \AA$. The mean value of the distances to the opposite oxygen atoms is $2.193 \mp 0.017 \AA$. The individual distances fall within the ranges $1.66-$ $1.86 \AA$ and $2.30-2.09 \AA$, respectively, but the deviations from the above mean values scarcely have statistical significance owing to the large standard deviations of the $z$ coordinates $(0.034-0.102 \AA$ for the oxygen atoms).

$\mathrm{Mo}_{7}$ is coordinated by seven oxygen atoms at the corners of a pentagonal bipyramid. The distances to the apical oxygen atoms discussed above are in this case 1.75 and $2.21 \AA$ ( \pm 0.04 $\AA)$. The distances to the remaining five oxygens situated at the corners of a pentagon are $1.94,2.03,2.00,2.02$ and $2.02 \AA( \pm 0.03 \AA)$ respectively. The corresponding five O-Mo-O angles subtended at the molybdenum atom by neighbouring oxygen atoms range from $70.0^{\circ}$ to $73.1^{\circ}\left( \pm 1.2^{\circ}\right)$. The oxygen-oxygen contacts within this pentagon remain rather close; they range from 2.26 to $2.41 \AA( \pm 0.04 \AA)$. All other oxygen-oxygen distances in the structure are greater than $2.47 \AA$.

The oxygen atom $\mathrm{O}_{20}$ is bound only to the molybdenum atom $\mathrm{Mo}_{8}$ and the separation, $1.67, \AA( \pm 0.04 \AA)$, is shorter by $0.08 \AA$ than the value previously obtained. It is in close agreement with the value of $1.671 \AA( \pm 0.008$ $\AA$ ) found for the distance between the molybdenum atom and the singly bonded oxygen atom in $\mathrm{MoO}_{3}{ }^{6}$.

Acknowledgements. I wish to thank Professor G. Hägg and Professor A. Magnéli for their continuous support. This work has been sponsored by the Swedish Natural Science Research Council. The computers BESK and FACIT EDB were used by permission of the Suedish Board for Computing Machinery.

1. Kihlborg, L. Acta Chem. Scand. 14 (1960) 1612.

2. Brändén, C.-I. and Lindqvist, I. Acta Chem. Scand. 17 (1963) 353.

3. Thomas, L. H. and Umeda, K. J. Chem. Phys. 26 (1957) 293.

4. Freeman, A. J. Acta Cryst. 12 (1959) 261.

5. Kihlborg, L. Arkiv Kemi. In press.

6. Kihlborg, L. Arkiv Kemi. In press.

Received June 8, 1963.
The Existence of Short- and Longchain Acyl-CoA:Carnitine Acyltransferases in Heart Mitochondria

\author{
KAARER. NORU M*
}

Institute of Clinical Biochemistry, Rikshospitalet, University of Oslo, Norway

$R$ esults have recently been obtained in this laboratory indicating that carnitine acts as a carrier of activated acyl groups through the mitochondrial membrane ${ }^{1-4}$. The acyl group is bound to carnitine by means of an ester linkage. The acylcarnitines were found to be formed according to the reaction: acyl-CoA + carnitine acylcarnitine $+\mathrm{CoA}$

and the reaction was found to be reversible.

It was suggested that acylation of carnitine with long-chain acyl groups is catalyzed by an enzyme which is different from the enzyme catalyzing the acylation of carnitine with short-chain acyl groups. The suggestion was based on the following criteria: (1) In the rat there are organ differences in the utilization of different acylcarnitines as measured by oxygen uptake $^{2}$. (2) There is no acetyl-CoA:carnitine acetyltransferase activity in the rat liver microsome fraction ${ }^{3}$, whereas this fraction has significant palmityl-CoA:carnitine palmityltransferase activity ${ }^{5}$. (3) Indications for a specific acetyl transfer enzyme were obtained when different $a$-keto acids were used as substrates for the mitochondrial acylation of carnitine ${ }^{4}$.

In the present communication results are reported which prove the existence of two different acyl-CoA:carnitine acyltransferases in ox heart mitochondria, one catalyzing the transfer of acetyl groups and the other catalyzing the transfer of palmityl groups.

Ox heart mitochondria were obtained as follows: The heart was removed immediately after death, cut into pieces and cooled on crushed ice. Fat and pericard were removed, and the heart muscle was homogenized in a Waring blendor in four volumes of $10 \%$ sucrose containing $5 \mathrm{mM}$ TRIS $(\mathrm{pH} 7.4)$ and $5 \mathrm{mM}$ EDTA. The mitochondria were isolated by differential centrifugation, washed once, and

\footnotetext{
* Fellow of the Norwegian Cancer Society.
}

Acta Chem. Scand. 17 (1963) No. 5 